\title{
Is physical activity a cause of longevity? It is not as straightforward as some would believe. $A$ critical analysis
}

\author{
Urho M Kujala
}

Faculty of Sport and Health Sciences, University of Jyväskylä, Jyväskylä, Finland

\section{Correspondence to}

Professor Urho M Kujala, Faculty of Sport and Health Sciences, University of Jyväskylä, Jyväskylä 40014, Finland: Urho.M.Kujala@jyu.fi

Accepted 13 February 2018 Published Online First 15 March 2018

- http://dx.doi.org/10.1136/ bjsports-2017-098995

- http://dx.doi.org/10.1136/ bjsports-2018-099185

Check for updates

To cite: Kujala UM.

Br J Sports Med

2018:52:914-918

\section{ABSTRACT}

There are discrepant findings between (A) observational follow-ups and (B) interventional studies that investigate possible causal association between high physical activity and low mortality. Participation in vigorous physical activity at a specific time-point is an indicator of good fitness and health, and is associated with a reduced risk of death. However, neither randomised controlled trials nor experimental animal studies have provided conclusive evidence to show that physical activity started during adulthood extends lifespan. Consequently, the undisputed health-related benefits of exercise have yet to translate into any proven causal relationship with longevity. Physical activity improves fitness and physical function, and confers other health-related effects. These outcomes have a greater basis in evidence-based data than any claims of a reduced risk of death, especially when recommending physical activity for previously physically inactive middle-aged and elderly adults.

The multiple benefits of exercise in terms of physical performance and health-related metabolism are incontrovertible. ${ }^{1-3}$ Exercise training (exercise therapy) has become an evidence-based component in the treatment and rehabilitation of chronic non-communicable disease, ${ }^{2}$ and plays a key role in maintaining or improving physical function, and in the prevention of disability. ${ }^{4}$ Substantial evidence exists to support the benefits of physical activity in terms of correcting many biological risk factor levels, potential harmful mechanisms are less studied, and robust data for mortality and lifespan are still missing.

A systematic review and meta-analysis of observational studies by Löllgen $e t \mathrm{al}^{5}$ reported that using 'mildly active' women as the reference, moderately active women had a $24 \%$ lower risk of death and the most active women had a $31 \%$ lower risk of death. The corresponding values for men were 19\% (advantage of moderately active over mildly active) and 24\% (advantage of most active over mildly active). The risk difference between the physical activity levels depends on how the cut-points for physical activity frequency, intensity or volume are set.

Among vigorously physically active, the magnitude of risk plateaus or turns U-shaped in very high volumes. ${ }^{67}$ Although the risk of death during exercise is low, the risk of sudden cardiac death is increased during episodic physical activity. ${ }^{8}$ Mittleman $e t a l^{9}$ found that the self-matched relative risk of myocardial infarction in the hour after heavy physical exertion (with an intensity of six or more metabolic equivalents), as compared with less strenuous physical exertion or none, was 5.9 (95\% CI 4.6 to 7.7$)$. Among people who usually exercised less than one, one to two, three to four, or five or more times per week, the respective relative risks were 107 (95\% CI 67 to 171), 19.4 (95\% CI 9.9 to 38.1$), 8.6(95 \%$ CI 3.6 to 20.5$)$ and $2.4(95 \%$ CI 1.5 to 3.7). Thus, increasing levels of habitual physical activity were associated with progressively lower relative risks of myocardial infarction during episodic vigorous physical activity. The induction time from heavy exertion to the onset of myocardial infarction was less than 1 hour, and symptoms usually began during the activity.

These observations illustrate the difference in the exercise-related risk of cardiac events among formerly physically inactive individuals compared with those having had long-term physically active lifestyle. Various contributory factors might be mechanistically related to sudden cardiac death, including coronary heart disease in older individuals, and a predominantly inherited and unsuspected cardiovascular disease among younger individuals. ${ }^{10}$ Plaque rupture related to exertion is one of the more unpredictable mechanistic causes for sudden death during or after physical exercise among individuals with coronary artery disease as well as in apparently healthy individuals. ${ }^{11} 12$

The aim of this paper is to critically evaluate the evidence used to recommend exercise-based lifestyle changes with the aim of increasing longevity. The discrepant interpretations of epidemiological associations versus intervention studies will be discussed, together with comment on the challenges to increase the level of evidence for studies in this field. Although this review is based on systematic literature searches, in addition to citing previous systematic reviews, the papers cited in this review were selected to illustrate the challenges in interpreting the associations between physical activity and mortality. The goal was not to provide an exhaustive list of original publications.

\section{EMERGENCE OF LONGEVITY}

Select individuals can survive to old age, even in populations with a relatively low mean life expectancy. ${ }^{13}$ In human and animal populations, a low mean life expectancy is associated with low lifespan equality. ${ }^{13}$ For these groups, reducing the number of premature deaths becomes a key factor in increasing the mean lifespan. Progress made in lengthening the human lifespan primarily reflects contemporary 


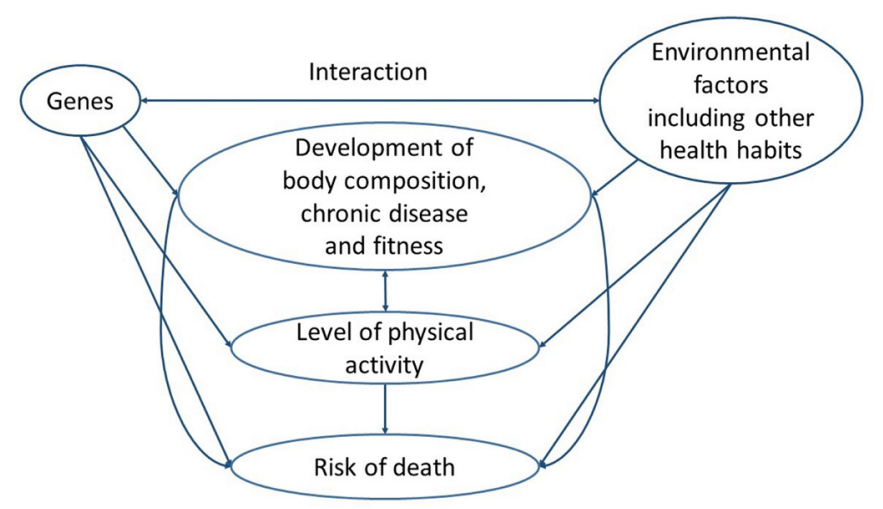

Figure 1 Factors that influence the association between physical activity and risk of death.

medical practice and public health efforts, combined with rising standards of living, superior education and healthier nutrition and lifestyles. ${ }^{14}$ The association between socioeconomic status and mortality is comparable in strength and consistency to that of tobacco use, alcohol consumption, insufficient physical activity, raised blood pressure, obesity and diabetes; smoking demonstrates the highest risk estimate. ${ }^{15}$

\section{CHALLENGES IN THE CORRECT INTERPRETATION OF OBSERVATIONAL DATA}

The classic London bus driver and conductor study showed that conductors suffered less coronary heart disease than drivers. ${ }^{16}$ The implication was that physical activity at work might prevent coronary heart disease in middle-aged men. However, in a follow-up paper, 'Physique of London busmen"17, epidemiological data for uniform size led to a different conclusion. This variable showed that drivers were larger than conductors, even when joining the service (ie, more obese individuals were preferentially becoming drivers at recruitment, thereby skewing the population characteristic). Consequently, selective differences in the driver/conductor populations, irrespective of physical activity, could have contributed to the differences found for coronary heart disease. Body composition, the early stages of undiagnosed disease and physical fitness all influence physical activity (figure 1). Although epidemiologists have done their best to take into account the confounding factors when analysing data and interpreting results, there are inevitable limitations in making conclusions for physical activity and causality. In humans, observational follow-up studies report an association between high baseline levels of leisuretime physical activity and low all-cause mortality. ${ }^{5}$ However, a causal relationship between adulthood physical activity and mortality/lifespan has neither been confirmed in randomised controlled intervention studies with initially healthy individuals $^{18} 19$ nor in animal experiments. ${ }^{19-21}$

Importantly, non-discretionary occupational physical activity is not consistently associated with a reduced risk of death. ${ }^{19}$ 22-25 There are selection issues likely explaining some of the difference between the associations between the voluntary leisure-time physical activity and occupational physical activity with mortality risk. However, there are also physiological differences between the most common types of leisure-time and occupational physical activity, ${ }^{26}$ which may partly explain the differences between the associations with mortality. When high fitness and good health are criteria for recruitment to a physically demanding occupation, there is a selection bias towards reduced mortality among those in physically demanding occupations compared with those in sedentary occupations or unemployed individuals.

On the basis of epidemiological estimates, physical inactivity is predicted to be causal in $9 \%$ of the cases of premature deaths worldwide, ${ }^{27}$ leading researchers to suggest that its elimination might increase life expectancy by 0.68 year, in line with similar estimates for smoking or obesity. ${ }^{27}$ However, we lack the intervention studies to make this assertion, with only observational evidence (ie, epidemiological associations) to link high levels of physical activity with a reduced risk of death later in life. ${ }^{18} 19$ In our twin study of monozygotic (MZ) twin pairs showing discordant patterns for smoking or physical activity, non-smoking showed a reduced risk for death unlike physical activity. ${ }^{28}$

When studying the role of physical activity versus inactivity in influencing lifespan, a key factor is whether the specific type of physical activity prevents or predisposes to premature death in an individual with specific biological characteristics. Further, many measureable and non-measureable lifestyle and individual biological factors are associated with a physically active lifestyle, with these serving to confound any evaluation of causation. ${ }^{18}$ Figure 1 shows how different variables may explain much of the observed association between baseline leisure-time physical activity and later mortality in epidemiological studies. Data from 38 original observational studies were included in the meta-analysis by Löllgen et al on physical activity and all-cause mortality. ${ }^{5}$ Most of the 38 included studies adjust for body mass index and many of them meritoriously adjust for socioeconomic status, smoking and apparent chronic diseases at baseline. However, the analyses are not adjusted for genes, physical fitness, detailed body composition measures or complex measures of social relationships and only some studies include adjustment for dietary factors. The association of social relationships on the risk of death has been reported to be comparable with the risk associated with smoking. ${ }^{29}$ Smoking is typically a 'health habit', which is less common among physically active individuals. When adjusting for smoking in epidemiological research it is also important to understand that among current smokers, the number of cigarettes smoked (ie, dose) is higher among those who are physically inactive versus their physically active counterparts, which contributes to the lower mortality of physically active individuals in observational studies.

\section{THE HEALTHY EXERCISER BIAS}

Contemporary researchers sometimes tend to analyse the data in an oversimplistic fashion and this can lead to erroneous interpretation of observational association studies. These include observations made by researchers for seniors. When healthy and relatively fit elderly individuals are able to participate in vigorous physical activity, and have an increased life expectancy compared with their less fit contemporaries (that fail to participate in vigorous physical activity), this has been interpreted by some researchers as providing evidence for a causal link between activity levels and a reduced risk of death. However, selection bias in the population is an alternative explanation. Aged individuals who are healthy enough (ie, not hampered by chronic disease) to exercise will manifest a reduced risk of death irrespective of their physical activity level. Consequently, studies which compare the predictive role of physical activity at younger versus older age show that older age physical activity is a stronger predictor of death. ${ }^{30-32}$ The consequence of optimistic 


\section{Observational follow-up study}

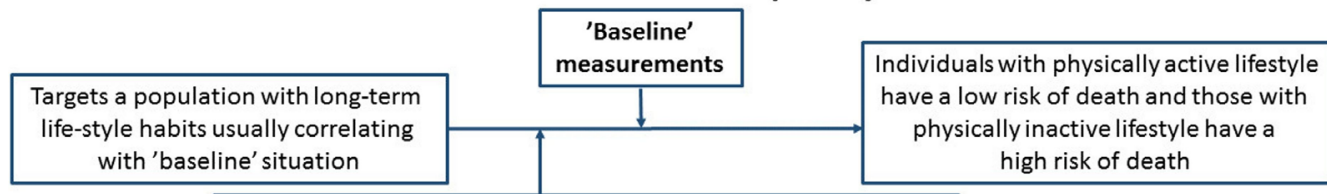

Note: Healthy and high-fitness individuals are able to increase their activity levels before 'baseline' and those with early-stage chronic disease have reduced their activity levels before 'baseline'

\section{Randomised controlled intervention trial (RCT)}

\begin{tabular}{|c|c|c|}
\hline \multicolumn{2}{|c|}{$\begin{array}{c}\text { Target population randomised } \\
\text { to exercise intervention and control groups }\end{array}$} \\
\hline $\begin{array}{c}\text { Usually targets a population sub-group } \\
\text { who has been physically inactive } \\
\text { during most of their adult life }\end{array}$ & \begin{tabular}{|l|l|l|l} 
In intervention group: Immediate increase in \\
physical activity, gradual increase in physical fitness, \\
possible gradual improvements in some other risk \\
factors, reduction in mortality? \\
In control group: No changes expected
\end{tabular} \\
\hline
\end{tabular}

Childhood

Older age

Figure 2 Differences between observational cohort studies and randomised controlled trials in investigating the relationships between physical activity and risk of death.

interpretation from observational associations is that older people are then encouraged to participate in physical activity to reduce mortality although confirmatory data from intervention studies are missing (figure 2 ). This practice may place some elderly individuals at risk for health complications should their recommended exercise not be properly tailored to their health status.

\section{PHYSICAL ACTIVITY, GENES, FITNESS AND THE RISK OF DEATH}

High cardiorespiratory fitness, as an integrated measure of body function, together with neuromuscular and metabolic function, is a strong predictor of reduced mortality in humans ${ }^{33-35}$ and in rats. ${ }^{19} 36$ Low cardiorespiratory fitness is associated with an increased risk of multiple chronic diseases and death. ${ }^{37}$ Although we need to keep in mind the possible challenges and differences in the accuracy of measuring activity versus fitness, aerobic fitness (maximal oxygen consumption, $\mathrm{VO}_{2} \max$ ) predicts mortality with greater accuracy than the level of physical activity when both are analysed together. ${ }^{38}$ This is particularly true for older individuals, when physical activity is recorded using scales for which the ability to exercise vigorously contributes to a physical activity classification. ${ }^{39}$ Thus, we lack definitive evidence for causality between physical activity and longevity.

To increase our understanding of the associations between genes, physical activity and mortality, we recently carried out two studies- one in rats and one in human twins. ${ }^{19}$ The first was a controlled intervention study on the effects of voluntary running during adulthood on lifespan. We used two rat strains, selectively bred for either low or high-intrinsic running capacity. The study confirmed that an inherited capacity for fitness increased longevity, but, unexpectedly, running reduced life expectancy in both rat strains.

In the second part of the study, ${ }^{19}$ we studied human twin pairs who were long-term discordant for vigorous physical activity (in 1975 and 1981), studying dizygotic (DZ) and MZ pairs separately. During follow-up (from 1981 to 2013) the unadjusted pairwise HR for death for the active compared with inactive members of the twin pairs among the $788 \mathrm{DZ}$ pairs was 0.58 (95\% CI 0.46 to 0.74$)$ and among the $231 \mathrm{MZ}$ pairs was 0.85 (95\% CI 0.56 to 1.30 ). Repeating this analysis in the baseline-healthy subgroup generated HRs of 0.64 (95\% CI 0.45 to 0.89 ) among DZ pairs and 1.05 (95\% CI 0.58 to 1.88 ) among $M Z$ pairs, respectively. High heritability of long-term moderate-to-vigorous physical activity reduced the number of $\mathrm{MZ}$ pairs discordant for physical activity and thus contributed to the only moderate statistical power in analysing pairwise risk differences among $M Z$ pairs.

Taken together, our results were consistent with the previous data collected for rodents ${ }^{40-42}$ and humans, ${ }^{43}$ which indicated that genetic predisposition plays a significant role in exercise participation. These results are also consistent with (1) our previous suggestion ${ }^{18} 33$ that genetic pleiotropy may partly explain the associations observed between high physical activity and mortality in epidemiological studies, and (2) the notion that inherited aerobic capacity is a predictor of longevity. ${ }^{336}$ Further studies in both animals and humans are now warranted if we are to determine whether increased aerobic fitness by training reduces the risk of death.

In the co-twin control studies of physical activity, discordance between the members of MZ twins in terms of long-term physical activity typically starts in adulthood. ${ }^{44}$ On the basis of our twin studies, ${ }^{1928}$ or studies in rodents, ${ }^{2045}$ physical activity that commences in adulthood (in twins after moving from childhood home environment or in full-grown animals) did not improve longevity. However, avoiding a sedentary state, prioritising a lower intensity of physical activity or starting physical activity at an earlier age may affect lifespan differently when compared with becoming more physically active later in adult life. ${ }^{4-47}$

Figure 2 demonstrates the hazards of interpreting data derived from an observational versus intervention study, highlighting the weakness of the former concerning the magnitude of effects. It might be that life expectancy is increased by being physically active early in life and maintaining this lifestyle throughout adulthood. However, it appears likely that estimates of improved longevity ${ }^{27}$ based on earlier epidemiological studies ${ }^{48}$ (ie, that inactive individuals would gain 
1.3-3.7 years from an age of 50 years by becoming active) may be overly optimistic extrapolations of the data. The ability to increase physical activity in later life is an indicator of reduced risk of death, but the causal relationship remains obscure.

\section{EVIDENCE FROM RANDOMISED CONTROLLED TRIALS}

Thus far, randomised controlled trials (RCT) have failed to provide conclusive evidence to show that exercise can extend lifespan/reduce premature deaths when individuals apparently healthy at baseline are randomised to physical activity/exercise versus control groups. Theoretically, it might be easier to detect an effect if a tailored exercise regime is analysed with respect to all-cause mortality using a target group of individuals at increased risk of death, such as the elderly, but there are no reports on trials with mortality as main outcome with appropriate statistical power calculation for this aim.

In the acclaimed and recently published LIFE Study of 1635 sedentary individuals aged $70-89$ years, ${ }^{50}$ an aerobically based, moderately intensive physical activity programme was not associated with reduced cardiovascular events although the intervention was effective in preventing mobility disability. Similarly, the Look AHEAD Study of 5145 overweight or obese patients with type 2 diabetes, ${ }^{51}$ including a combination of increased physical activity and improved diet intervention, reported a difference in fitness between the intervention and control groups, but no difference in cardiovascular events. Even longer follow-ups may be needed to document the effects of exercise and lifestyle interventions on mortality as a statistically significant difference in mortality between the combined lifestyle intervention groups (diet, exercise or both) compared with control group in the cluster randomised Da Qing Diabetes Prevention Study targeting individuals with impaired glucose tolerance was not seen in the 20-year follow-up but was seen first in the 23 -year follow-up $(p=0.049) .5253$

Based on experience, clinical exercise testing might help determine safe exercise intensities, and identify when exercise could provoke findings or symptoms. This testing may then lead to tailored exercise recommendations, tweaks to medical treatment or even operative intervention. This approach, expected to lead to a decreased risk of death, is commonly practised in well-planned RCTs for cardiac patients. However, interestingly, a statistically significant reduction in all-cause mortality has not been seen in the latest and largest meta-analyses inclusive of exercise-based cardiac rehabilitation for coronary heart disease (meta-analysis including 47 studies with 12455 participants; pooled risk ratio for intervention compared with control groups 0.96 (95\% CI 0.88 to 1.04$))^{54}$ and exercise-based rehabilitation for heart failure (meta-analysis including 25 trials with 1871 participants; pooled risk ratio for intervention compared with control groups 0.93 (95\% CI 0.69 to 1.27$)) .{ }^{55}$ Also, in the large heart failureaction $\operatorname{trial}^{56}(\mathrm{n}=2331)$ there was no statistically significant reduction in the non-adjusted analysis of all-cause mortality (HR $0.96,95 \%$ CI 0.79 to $1.17 ; p=0.70$ ). While select trials have reported long-term follow-up results with a tendency towards reduced all-cause mortality in exercise intervention groups compared with controls, the issue of potential publication bias is difficult to analyse in these scenarios. Therefore, based on the current evidence, exercise-based rehabilitation for cardiac conditions cannot be recommended to reduce all-cause mortality, although it can be recommended in terms of other health benefits.

\section{CHALLENGES TO MOVING FORWARD}

Observational studies do not provide definitive answers on the causal relationship between physical activity and mortality. Including objective recording of physical activity to cohort studies increases the accuracy of documenting activity levels, but does not tackle the main problems of observational studies, such as selection issues related to genetic pleiotropy. It can be expected that individuals who do not have physical activity limitations due to early stages of chronic disease or due to naturally low fitness are more physically active both during leisure-time and in identical physically demanding occupations.

The author is not aware of appropriately randomised and statistically powered real-life trials with a younger target group and mortality as main outcome, perhaps because such trials are not feasible due to the unrealistically large sample sizes and long follow-up required. As it has been challenging to show the effects of combined diet and exercise intervention on mortality in middle-aged to older samples using RCT designs, it may also be difficult, but not impossible, for exercise interventions. In younger age groups the deaths are rare, and issues related to voluntariness and health screening of participants as well as possible cointerventions in exercise groups usually make generalisation of results challenging towards general recommendations for the elderly populations. However, the results of the promising Generation 100 Study $^{57}$ which has randomised 1567 selected (22\% of invited participants randomised) older individuals to exercise training versus control groups with the primary aim to evaluate the effects of 5 years of exercise training on mortality will be of interest.

Carefully planned, collaborative individual participant data (IPD) meta-analyses ${ }^{58}$ of RCTs might provide additional information. Also concerning IPD meta-analyses the challenge is how to get all relevant trials included and how to interpret the results from trials with carefully tailored clinical baseline examinations and selected volunteers to real-life settings. In clinical medical praxis an important target group

\section{Key messages}

On the basis of observational population studies, high physical activity is associated with a reduced risk of premature death, but causal relationship remains unproven.

- Participation in physical activity at a specific time-point is an indicator of good fitness and health, and is associated with a reduced risk of death.

- At present, there are no definitive data with which to draw final conclusions for the effect of adulthood physical activity on lifespan, either from randomised controlled trials or experimental animal studies.

- Lifelong physical activity may extend lifespan, but evidence from interventional studies is limited to some suggestive findings; the potential effects of (early) childhood physical activity versus inactivity on later health needs more research.

- The possible life-shortening effects of vigorous physical activity are understudied.

- Physical activity improves fitness and physical function, and confers other health-related benefits. These outcomes have a greater basis in terms of evidence-based data than any claims of a reduced risk of death, especially when recommending physical activity for previously physically inactive middle-aged and elderly adults. 
is those individuals who usually do not volunteer to any clinical trials ${ }^{59-61}$ and data on the effects of increasing physical activity on the risk of death are missing. Also it is difficult to generalise the results of randomised trials to patients with many coexisting diseases. ${ }^{2}$ Usually, also the control groups of participants of RCTs have lower risk of death than the patient groups overall. So, novel approaches, such as carefully tailored Benchmarking Controlled Trials, ${ }^{62}$ may be needed to produce data generalisable to clinical praxis concerning increasing physical activity and risk of death.

Further, collaborative co-twin control studies among MZ twin pairs with larger sample sizes through international collaboration and long-term follow-ups with appropriate baseline examinations might be valuable. However, it needs to be kept in mind that physical activity discordances among DZ pairs may occur due to genetic differences and only findings from MZ pairs fully control for genes at sequence level. Mendelian randomisation analyses ${ }^{63}$ and studies on genetic pleiotropy ${ }^{64}$ might also help in clarifying the role of genetic factors, and then interpreting correctly the discrepant findings from observational and interventional studies. Researchers have also tried to develop useful biomarkers of ageing to investigate which factors play a role in accelerating or decelerating the ageing process. Proposed biomarkers include measuring telomere length ${ }^{65}$ and the DNA methylation age (epigenetic clock). ${ }^{66}$ Shortening of telomeres that induces cell senescence or apoptosis is associated with shortened life expectancy. Again, based on a recent meta-analysis, no consistent association has been found between physical activity and telomere length. ${ }^{65}$ Our studies in MZ twin pair long-term discordant for physical activity do show differences neither in telomere length nor in epigenetic clock (unpublished).

We need ambitious researchers to design studies, which in future will clarify the elusive relationship between physical activity and mortality. Meanwhile, in our medical clinical practice, it is better to recommend exercise for the more evidencebased reasons. ${ }^{2}{ }^{3}$ 'Healthier' life is an important outcome in the presence or absence of increased life expectancy.

Funding The study was supported by the Finnish Ministry of Education and Culture (grant: OKM/56/626/2013).

Competing interests None declared.

Patient consent Not required.

Provenance and peer review Not commissioned; externally peer reviewed.

(c) Article author(s) (or their employer(s) unless otherwise stated in the text of the article) 2018. All rights reserved. No commercial use is permitted unless otherwise expressly granted.

\section{REFERENCES}

1 Physical Activity Guidelines Advisory Committee. Physical activity guidelines advisory committee report. Washington, DC: U.S: Department of Health and Human Services, 2008.

2 Kujala UM. Evidence on the effects of exercise therapy in the treatment of chronic disease. Br J Sports Med 2009:43:550-5.

3 Pedersen BK, Saltin B. Exercise as medicine - evidence for prescribing exercise as therapy in 26 different chronic diseases. Scand J Med Sci Sports 2015;25(Suppl. 3): $1-72$.

4 Pasanen T, Tolvanen S, Heinonen A, et al. Exercise therapy for functional capacity in chronic diseases: an overview of meta-analyses of randomised controlled trials. $\mathrm{Br}$ Sports Med 2017;51:1459-65.

5 Löllgen H, Böckenhoff A, Knapp G. Physical activity and all-cause mortality: an updated meta-analysis with different intensity categories. Int I Sports Med 2009:30:213-24

6 O'Keefe JH, Patil HR, Lavie CJ, et al. Potential adverse cardiovascular effects from excessive endurance exercise. Mayo Clin Proc 2012;87:587-95.

7 Eijsvogels TM, Molossi S, Lee DC, et al. Exercise at the extremes: the amount of exercise to reduce cardiovascular events. J Am Coll Cardiol 2016;67:316-29.
8 Dahabreh IJ, Paulus JK. Association of episodic physical and sexual activity with triggering of acute cardiac events: systematic review and meta-analysis. JAMA 2011;305:1225-33.

9 Mittleman MA, Maclure M, Tofler GH, et al. Triggering of acute myocardial infarction by heavy physical exertion -- protection against triggering by regular exertion. $N$ Engl J Med Overseas Ed 1993:329:1677-83.

10 Maron BJ, Doerer JJ, Haas TS, et al. Sudden deaths in young competitive athletes: analysis of 1866 deaths in the United States, 1980-2006. Circulation 2009;119:1085-92.

11 Ciampricotti R, el-Gamal M, Relik T, et al. Clinical characteristics and coronary angiographic findings of patients with unstable angina, acute myocardial infarction, and survivors of sudden ischemic death occurring during and after sport. Am Heart J 1990;120:1267-78.

12 Burke AP, Farb A, Malcom GT, et al. Plaque rupture and sudden death related to exertion in men with coronary artery disease. JAMA 1999;281:921-6.

13 Colchero F, Rau R, Jones OR, et al. The emergence of longevous populations. Proc Natl Acad Sci U S A 2016;113:E7681-90.

14 Vaupel JW. Biodemography of human ageing. Nature 2010;464:536-42.

15 Stringhini S, Carmeli C, Jokela M, et al. Socioeconomic status and the $25 \times 25$ risk factors as determinants of premature mortality: a multicohort study and meta-analysis of 1.7 million men and women. Lancet 2017;389:1229-37.

16 Morris JN, Heady JA, Raffle PA, et al. Coronary heart-disease and physical activity of work. Lancet 1953;265:1053-7.

17 Heady JA, Morris JN, Raffle PA. Physique of London busmen; epidemiology of uniforms. Lancet 1956;271:569-70.

18 Kujala UM. Physical activity, genes, and lifetime predisposition to chronic disease. European Review of Aging and Physical Activity 2011;8:31-6.

19 Karvinen S, Waller K, Silvennoinen $\mathrm{M}$, et al. Physical activity in adulthood: genes and mortality. Sci Rep 2015;5:18259.

20 Goodrick CL, Ingram DK, Reynolds MA, et al. Differential effects of intermittent feeding and voluntary exercise on body weight and lifespan in adult rats. J Gerontol 1983:38:36-45.

21 Garcia-Valles R, Gomez-Cabrera MC, Rodriguez-Mañas L, et al. Life-long spontaneous exercise does not prolong lifespan but improves health span in mice. Longev Healthspan 2013:2:14.

22 Paffenbarger RS, Laughlin ME, Gima AS, et al. Work activity of longshoremen as related to death from coronary heart disease and stroke. $N$ Engl I Med 1970;282:1109-14.

23 Samitz G, Egger M, Zwahlen M. Domains of physical activity and all-cause mortality: systematic review and dose-response meta-analysis of cohort studies. Int J Epidemiol 2011:40:1382-400.

24 Holtermann A, Marott JL, Gyntelberg F, et al. Occupational and leisure time physical activity: risk of all-cause mortality and myocardial infarction in the Copenhagen City Heart Study. A prospective cohort study. BMJ Open 2012;2:e000556.

25 Richard A, Martin B, Wanner M, et al. Effects of leisure-time and occupational physical activity on total mortality risk in NHANES III according to sex, ethnicity, central obesity, and age. J Phys Act Health 2015:12:184-92.

26 Holtermann A, Krause N, van der Beek AJ, et al. The physical activity paradox: six reasons why occupational physical activity (OPA) does not confer the cardiovascular health benefits that leisure time physical activity does. $\mathrm{Br}$ I Sports Med 2018;52:149-50.

27 Lee IM, Shiroma EJ, Lobelo F, et al. Effect of physical inactivity on major noncommunicable diseases worldwide: an analysis of burden of disease and life expectancy. Lancet 2012;380:219-29.

28 Kujala UM, Kaprio J, Koskenvuo M. Modifiable risk factors as predictors of allcause mortality: the roles of genetics and childhood environment. Am J Epidemiol 2002;156:985-93.

29 Holt-Lunstad J, Smith TB, Layton JB. Social relationships and mortality risk: a metaanalytic review. PLoS Med 2010;7:e1000316.

30 Bijnen FC, Feskens EJ, Caspersen CJ, et al. Baseline and previous physical activity in relation to mortality in elderly men: the Zutphen Elderly Study. Am J Epidemiol 1999;150:1289-96.

31 Sherman SE, D'Agostino RB, Silbershatz $\mathrm{H}$, et al. Comparison of past versus recent physical activity in the prevention of premature death and coronary artery disease. Am Heart J 1999:138:900-7.

32 Keadle SK, Arem H, Moore SC, et al. Impact of changes in television viewing time and physical activity on longevity: a prospective cohort study. Int I Behav Nutr Phys Act 2015;12:156.

33 Kujala UM, Marti P, Kaprio J, et al. Occurrence of chronic disease in former top-level athletes. Sports Medicine 2003;33:553-61.

34 Myers J, Prakash M, Froelicher V, et al. Exercise capacity and mortality among men referred for exercise testing. N Engl J Med 2002;346:793-801.

35 Kodama S, Saito K, Tanaka S, et al. Cardiorespiratory fitness as a quantitative predicto of all-cause mortality and cardiovascular events in healthy men and women: a metaanalysis. JAMA 2009;301:2024-35.

36 Koch LG, Kemi OJ, Qi N, et al. Intrinsic aerobic capacity sets a divide for aging and longevity. Circ Res 2011;109:1162-72. 
37 Ross R, Blair SN, Arena R, et al. Importance of Assessing Cardiorespiratory Fitness in Clinical Practice: A Case for Fitness as a Clinical Vital Sign: A Scientific Statement From the American Heart Association. Circulation 2016;134:e653-99.

38 Lee DC, Sui X, Ortega FB, et al. Comparisons of leisure-time physical activity and cardiorespiratory fitness as predictors of all-cause mortality in men and women. $\mathrm{Br} J$ Sports Med 2011;45:504-10.

39 Äijö M, Kauppinen M, Kujala UM, et al. Physical activity, fitness, and all-cause mortality: An 18-year follow-up among old people. J Sport Health Sci 2016;5:437-42.

40 Koch LG, Britton SL, Wisløff U. A rat model system to study complex disease risks, fitness, aging, and longevity. Trends Cardiovasc Med 2012;22:29-34.

41 Kelly SA, Nehrenberg DL, Peirce JL, et al. Genetic architecture of voluntary exercise in an advanced intercross line of mice. Physio/ Genomics 2010;42:190-200.

42 Novak CM, Escande C, Burghardt PR, et al. Spontaneous activity, economy of activity, and resistance to diet-induced obesity in rats bred for high intrinsic aerobic capacity. Horm Behav 2010;58:355-67.

43 Stubbe JH, Boomsma DI, Vink JM, et al. Genetic influences on exercise participation in 37,051 twin pairs from seven countries. PLoS One 2006;1:e22.

44 Rottensteiner M, Leskinen T, Niskanen E, et al. Physical activity, fitness, glucose homeostasis, and brain morphology in twins. Med Sci Sports Exerc 2015;47:509-18.

45 Edington DW, Cosmas AC, McCafferty WB. Exercise and longevity: evidence for a threshold age. J Gerontol 1972;27:341-3.

46 Goodrick CL. Effects of long-term voluntary wheel exercise on male and female Wistar rats. I. Longevity, body weight, and metabolic rate. Gerontology 1980;26:22-33.

47 Holloszy JO, Smith EK, Vining M, et al. Effect of voluntary exercise on longevity of rats. J Appl Physiol 1985;59:826-31.

48 Paffenbarger RS, Hyde RT, Wing AL, et al. Physical activity, all-cause mortality, and longevity of college alumni. N Engl J Med 1986;314:605-13.

49 Franco $\mathrm{OH}$, de Laet $\mathrm{C}$, Peeters $\mathrm{A}$, et al. Effects of physical activity on life expectancy with cardiovascular disease. Arch Intern Med 2005;165:2355-60.

50 Newman AB, Dodson JA, Church TS, et al. Cardiovascular events in a physical activity intervention compared with a successful aging intervention: the life study randomized trial. JAMA Cardiol 2016;1:568-74.

51 Wing RR, Bolin P, Brancati FL, et al. Cardiovascular effects of intensive lifestyle intervention in type 2 diabetes. N Eng/ J Med 2013;369:145-54.

52 Li G, Zhang P, Wang J, et al. The long-term effect of lifestyle interventions to prevent diabetes in the China Da Qing Diabetes Prevention Study: a 20-year follow-up study. Lancet 2008;371:1783-9.
53 Li G, Zhang P, Wang J, et al. Cardiovascular mortality, all-cause mortality, and diabetes incidence after lifestyle intervention for people with impaired glucose tolerance in the Da Qing Diabetes Prevention Study: a 23-year follow-up study. Lancet Diabetes Endocrinol 2014;2:474-80.

54 Anderson L, Oldridge N, Thompson DR, et al. Exercise-based cardiac rehabilitation for coronary heart disease: cochrane systematic review and meta-analysis. J Am Coll Cardiol 2016;67:1-12.

55 Sagar VA, Davies EJ, Briscoe $S$, et al. Exercise-based rehabilitation for heart failure: systematic review and meta-analysis. Open Heart 2015;2:e000163.

56 O'Connor CM, Whellan DJ, Lee KL, et al. Efficacy and safety of exercise training in patients with chronic heart failure: HF-ACTION randomized controlled trial. JAMA 2009;301:1439-50.

57 Stensvold D, Viken H, Rognmo Ø, et al. A randomised controlled study of the longterm effects of exercise training on mortality in elderly people: study protocol for the Generation 100 study. BMJ Open 2015;5:e007519.

58 Riley RD, Lambert PC, Abo-Zaid G. Meta-analysis of individual participant data: rationale, conduct, and reporting. BMJ 2010;340:c221.

59 Lerman Y, Shemer J. Epidemiologic characteristics of participants and nonparticipants in health-promotion programs. J Occup Environ Med 1996;38:535-8.

60 Lakerveld J, ljzelenberg W, van Tulder MW, et al. Motives for (not) participating in a lifestyle intervention trial. BMC Med Res Methodol 2008:8:17.

61 Gesche J, Renault K, Nørgaard K, et al. Representativeness of participants in a lifestyle intervention study in obese pregnant women - the difference between study participants and non-participants. Obes Facts 2014;7:351-60.

62 Malmivaara A. Assessing validity of observational intervention studies - the Benchmarking Controlled Trials. Ann Med 2016;48:440-3.

63 Latvala A, Ollikainen M. Mendelian randomization in (epi)genetic epidemiology: an effective tool to be handled with care. Genome Biol 2016;17:156.

64 Visscher PM, Wray NR, Zhang Q, et al. 10 years of GWAS discovery: biology, function, and translation. Am J Hum Genet 2017;101:5-22.

65 Mundstock E, Zatti H, Louzada FM, et al. Effects of physical activity in telomere length: Systematic review and meta-analysis. Ageing Res Rev 2015;22:72-80.

66 Horvath S. DNA methylation age of human tissues and cell types. Genome Biol 2013:14:R115 